\title{
Clinical and radiological features related to the growth potential of meningioma
}

\author{
Hidetoshi Kasuya • Osami Kubo - Masahiko Tanaka • \\ Kosaku Amano • Koichi Kato • Tomokatsu Hori
}

Received: 9 March 2006 / Revised: 13 April 2006 / Accepted: 28 May 2006 / Published online: 5 September 2006

(C) Springer-Verlag 2006

\begin{abstract}
Clinical and radiological features that help predict the growth potential of meningioma would be beneficial. The purpose of this study is to clarify the characteristics related to proliferating potential using the MIB-1 staining index. We analyzed the relationship of MIB-1 staining indices to characteristics of 342 consecutive patients with meningioma surgically removed between 1995 and 2004 by logistic regression analysis. One hundred and forty-nine of the patients with meningioma were $\geq 60$ in age; 89 male; 48 recurrent; 203 symptomatic; 157 at the skull base; 124 over $20 \mathrm{~cm}^{3} ; 24$ multiple; 136 with edema; 117 with calcification. The MIB-1 staining index in 56 of 296 grade $I$ meningiomas in WHO classification was $\geq 3.0$; in 27 of 28 grade II; and in 17 of 18 grade III, respectively. Logistic regression analysis demonstrated that male (odds ratio [OR], 2.374, $\mathrm{p}=0.003)$, recurrence (OR, 7.574, $\mathrm{p}=0.0001$ ), skull base (OR, 0.540, $\mathrm{p}=0.035$ ), calcification (OR, 0.498, $\mathrm{p}=0.019$ ) were independent risk factors for a high MIB-1 staining index $(\geq 3.0)$; age, symptomatic, volume, multiple, edema were not. Male, recurrence, non-skull base, absence of calcification are independent risk factors for a high proliferative potential. These should be taken into consideration when managing meningiomas.
\end{abstract}

Keywords Growth $\cdot$ Meningioma $\cdot$ MIB-1

H. Kasuya $(\bowtie) \cdot$ O. Kubo $\cdot$ M. Tanaka $\cdot$ K. Amano $\cdot$ K. Kato $\cdot$

T. Hori

Department of Neurosurgery,

Tokyo Women's Medical University,

Kawada-cho 8-1, Shinjyuku-ku,

Tokyo 162-8666, Japan

e-mail: hkasuya@nij.twmu.ac.jp

\section{Introduction}

An estimated $2-3 \%$ of the population has an incidental asymptomatic meningioma in autopsy studies $[14,20]$. With the wider use of CT and MRI, many meningiomas are discovered as incidental findings during investigation for unrelated symptoms $[9,13,16,17,20,21]$. The growth potential of meningiomas varies. Some meningiomas remain unchanged in size for a long period, whereas others grow rapidly [15]. Sex, age, initial tumor size, and calcification were reported to be related to the tumor growth judging from follow-up scans [9, 13, 16, 17, 21].

The nuclear antigen Ki-67 expressed by proliferating cells has become available for routinely processed paraffin section. The MIB-1 antibody detects an epitope on the Ki67 antigen, a nuclear protein present only during active phase of the cell cycle (G1, S, G2, and M) [2]. Several studies investigated how Ki-67 labelling indices could help to predict recurrence $[1,3,4,6-8,11,12,18,19]$. An increased MIB-1 staining index was highly correlated with a shorter tumor volume doubling time [12]. In the cases that showed an MIB-1 of $\geq 3 \%$, the tumor volume doubling time was $<2$ years. Nakaguchi et al. [12] found the formula which can calculate tumor doubling time (Td) from the MIB-1 staining index at surgery: $\log \mathrm{Td}=31.4-0.14 \times \mathrm{MIB}-1$ SI $\left(\mathrm{R}^{2}=0.556\right)$. The time interval to the next recurrence for recurrent meningiomas is associated with the MIB-1 staining index. Meningiomas with MIB-1 staining index of $3 \%$ or higher had a significantly higher tendency of recurrence [11]. Although these cell kinetics methods are valuable for growth potential, they can be applied only after the verification of pathology [15].

The purpose of this study was to clarify the clinical and radiological features related to meningioma proliferation using the MIB-1 staining index. Sex, age, calcification, 
edema, symptom, size, and shape of tumor were already reported to be related to the tumor growth and MIB-1 staining index $[1,3,4-8,11-13,16-19,21]$. The results, however, were inconsistent because of a lack of sufficient case number and inadequate statistical analysis. Independent risk factors for high growth rate should be determined. We analyzed the relationship of MIB-1 staining indices to the characteristics of 342 consecutive patients with meningioma surgically removed between 1995 and 2004 by logistic regression analysis.

\section{Materials and methods}

Three hundred and forty-two patients with meningiomas were surgically treated in our department of neurosurgery between 1995 and 2004. Radiological features were analyzed by CT scans and/or MRI. Location of the tumor was classified as follows: convexity, falx, parasagittal, sylvian fissure, tentorial, ventricular, foramen magnum, olfactory groove, petroclival, petrous, sphenoid ridge, and tuberculum sellae. The latter six locations were considered as skull base. The tumor volume was calculated using the formula: length $\times$ depth $\times$ width $\times 0.5$ [9]. When patients had multiple meningiomas, only the largest tumor was included. On the basis of conventional CT and bone window $\mathrm{CT}$, patients were divided into two groups according to the low density area around the tumor and calcification in the tumor. A low density area due to surgical scar was not included in edema in patients with recurrent meningioma.

The tumors were histologically classified according to the World Health Organization classification of tumors [10]. An avidin-biotin immunoperoxidase or simple stain MAX-peroxidase (Nichirei, Tokyo) technique was used to perform MIB-1 monoclonal antibody (DAKO, Denmark) assay in selected sections of each case. All tissue sections were examined at high-power magnification $(\times 400)$. The number of cells stained positively with MIB-1 and the total number of tumor cells were counted in several representative fields containing more than 1,000 cells. Their ratio was indicated as the MIB-1 staining index (\%).

\section{Statistical analysis}

All data were stored on a personal computer and analyzed using commercially available statistical software (SPSS version 12.0, SPSS Inc.). Chi-squired analysis was used to compare the MIB-1 staining index to characteristics of patients with meningioma. All variables were included in a logistic regression model to determine which variables were independently associated with a high MIB-1 staining index
( $\geq 3.0$ ). Significance was judged at a value of $\mathrm{p}<0.05$ for all analyses.

\section{Results}

Table 1 shows the characteristics and MIB-1 staining index of the 342 patients. One hundred and forty-nine of patients with meningioma were $\geq 60$ in age; 89 male; 48 recurrent; 203 symptomatic; 157 at the skull base; $124 \geq 20 \mathrm{~cm}^{3}$ in volume; 24 multiple; 136 with edema; 117 with calcification. We compare these characteristics to the MIB-1 staining index. We divided them into two groups: $<3.0$ and $\geq 3.0$ [11]. The MIB-1 staining index in 100 of 342 meningiomas was $>3.0$. Sex $(p=0.0001)$, recurrence $(p=0.0001)$, symptomatic $(p=0.013)$, volume $(p=0.014)$, edema $(p=0.001)$, and calcification $(\mathrm{p}=0.0001)$ were correlated with the MIB-1 staining index by chi-square test; age, skull base, and multiple were not.

Meningothelial, transitional, and fibrous meningiomas were the three major subtypes, and they accounted for about three fourth of the total. Two hundred and ninety-six meningiomas belonged to grade I; 28 grade II; and 18 grade III. MIB-1 staining index in 56 of 296 grade I meningiomas was $\geq 3.0$; that in 27 of 28 grade II; and that in 17 of 18 grade $I I I$, respectively (Table 2 ).

Logistic regression analysis demonstrated that male (odds ratio [OR], 2.374, $\mathrm{p}=0.003$ ), recurrence (OR, 7.574, $\mathrm{p}=0.0001)$, skull base $(\mathrm{OR}, 0.540, \mathrm{p}=0.035)$, calcification (OR, 0.498, $\mathrm{p}=0.019)$ were independent risk factors for a

Table 1 Characteristics and MIB-1 staining index in 342 meningiomas

\begin{tabular}{llll}
\hline & \multicolumn{2}{l}{ MIB-1 staining index (\%) } & \\
\cline { 2 - 3 } Factor & $<3.0$ & $\geq 3.0$ & P Value \\
\hline Age (years) & & & 0.937 \\
-49 & 65 & 24 & \\
$50-$ & 72 & 32 & \\
$60-$ & 79 & 34 & \\
$70-$ & 26 & 10 & \\
Sex (male/female) & $46 / 196$ & $43 / 57$ & 0.0001 \\
Recurrence (yes/no) & $14 / 228$ & $34 / 66$ & 0.0001 \\
Symptomatic (yes/no) & $133 / 109$ & $70 / 30$ & 0.013 \\
Skull base (yes/no) & $119 / 123$ & $38 / 62$ & 0.059 \\
Volume (cm ${ }^{3}$ ) & & & 0.014 \\
-9.9 & 113 & 37 & \\
$10-$ & 53 & 15 & \\
$20-$ & 76 & 48 & \\
Multiple (yes/no) & $15 / 227$ & $9 / 91$ & 0.244 \\
Edema (yes/no) & $82 / 160$ & $54 / 46$ & 0.001 \\
Calcification (yes/no) & $97 / 145$ & $20 / 80$ & 0.0001 \\
Total & 242 & 100 & \\
\hline
\end{tabular}


Table 2 Histological subtypes and MIB-1 staining index of 342 meningiomas

\begin{tabular}{|c|c|c|c|}
\hline \multirow[t]{2}{*}{ Subtype } & \multicolumn{2}{|c|}{ MIB-1 staining index (\%) } & \multirow[t]{2}{*}{ Total } \\
\hline & $<3.0$ & $\geq 3.0$ & \\
\hline \multicolumn{4}{|l|}{ Grade I } \\
\hline Meningothelial & 106 & 28 & 134 \\
\hline Fibrous & 67 & 13 & 80 \\
\hline Transitional & 43 & 13 & 56 \\
\hline Psammomatous & 6 & 0 & 6 \\
\hline Angiomatous & 13 & 2 & 15 \\
\hline Microcystic & 1 & 0 & 1 \\
\hline Secretory & 1 & 0 & 1 \\
\hline Lymphoplasmacyte-rich & 1 & 0 & 1 \\
\hline \multirow[t]{2}{*}{ Metaplastic } & 2 & 0 & 2 \\
\hline & 240 & 56 & 296 \\
\hline \multicolumn{4}{|l|}{ Grade II } \\
\hline Atypical & 1 & 26 & 27 \\
\hline \multirow[t]{2}{*}{ Chordoid } & 0 & 1 & 1 \\
\hline & 1 & 27 & 28 \\
\hline \multicolumn{4}{|l|}{ Grade III } \\
\hline Rhabdoid & 0 & 1 & 1 \\
\hline Papillary & 1 & 1 & 2 \\
\hline \multirow[t]{2}{*}{ Anaplastic } & 0 & 15 & 15 \\
\hline & 1 & 17 & 18 \\
\hline Total & 242 & 100 & 342 \\
\hline
\end{tabular}

high MIB-1 staining index ( $\geq 3.0)$; age, symptomatic, volume, multiple, and edema were not (Table 3 ).

\section{Discussion}

We analyzed the relationship of the MIB-1 staining indices to the characteristics of 342 consecutive patients with meningioma surgically removed between 1995 and 2004 by logistic regression analysis. Logistic regression analysis demonstrated that male (odds ratio [OR], 2.374, $\mathrm{p}=0.003$ ), recurrence $(\mathrm{OR}, 7.574, \mathrm{p}=0.0001)$, skull base $(\mathrm{OR}, 0.540$, $\mathrm{p}=0.035)$, calcification $(\mathrm{OR}, 0.498, \mathrm{p}=0.019)$ were indepen-

Table 3 Logistic regression analysis for factors independently related to MIB-1 staining index

\begin{tabular}{llll}
\hline Factor & Odds ratio & $95 \% \mathrm{CI}$ & P Value \\
\hline Age & 1.109 & $0.841-1.461$ & 0.464 \\
Sex & 2.374 & $1.336-4.219$ & 0.003 \\
Recurrence & 7.574 & $3.558-16.124$ & 0.0001 \\
Symptomatic & 1.468 & $0.774-2.784$ & 0.240 \\
Skull base & 0.540 & $0.305-0.956$ & 0.035 \\
Volume & 1.332 & $0.944-1.879$ & 0.103 \\
Multiple & 1.027 & $0.398-2.651$ & 0.957 \\
Edema & 1.508 & $0.838-2.711$ & 0.170 \\
Calcification & 0.498 & $0.278-0.892$ & 0.019 \\
\hline
\end{tabular}

dent risk factors for a high MIB-1 staining index $(\geq 3.0)$; age, symptomatic, volume, multiple, and edema were not.

The relationship between the growth rate or MIB-1 staining index and age has been controversial: a higher MIB-1 staining index and higher growth rate were observed for younger patients $[11,13,21]$; but not in other reports $[1$, $12,15,19]$. Our series of 342 patients with meningioma showed no relation.

It is well known that atypical and anaplastic meningiomas are predominant in males [10]. Matsuno et al. [11] reported that the mean MIB-1 staining index in 50 male patients was $5.5 \%$, whereas that in 77 female patients was $2.7 \%$. Our findings show that male (odds ratio [OR], 2.374, $\mathrm{p}=0.003$ ) was an independent risk factor for a high MIB-1 staining index. We also found a higher MIB-1 staining index in males even in grade I meningioma (MIB-1 staining index in 32 of 226 females, and in 24 of 70 males was $\geq 3.0, \mathrm{p}=0.0001$, chi-squire test).

Recurrence (OR, 7.574, $\mathrm{p}=0.0001)$ was the most significant independent risk factor for a high MIB-1 staining index $(\geq 3.0)$. Therefore, we propose prompt management for recurrent meningiomas. In most of the recurrent meningiomas, the MIB-1 staining index was higher at the time of recurrence than at the time of initial surgery $[1,11,19]$. Changes in histological morphology and malignant transformation are also known in meningiomas.

Although there is a significant difference in the MIB-1 staining index between symptomatic and non-symptomatic meningiomas by chi-square test, symptomatic is not an independent risk factor for a high MIB-1 staining index. Meningiomas commonly present with seizure disorders, and are associated with location, perilesional edema, and convexity location. Symptoms and signs of elevated intracranial pressure could be due to the large size of meningioma itself, or to the pronounced cerebral swelling resulting from reactive vasogenic edema. Focal neurological deficits caused by meningiomas are generally related to direct local brain, cranial-nerve compression, and can be predicted from the site of origin of the tumor [20]. Thus, symptomatic meningioma may not be related to a high MIB-1 staining index. The growth rate of incidental meningioma may be similar to that of symptomatic meningioma.

Our results demonstrated that skull base (OR, 0.540, $\mathrm{p}=0.035)$ is an independent risk factor for a high MIB-1 staining index. No relationship has been reported between the MIB-1 staining index and the location of meningiomas $[15,19]$. In general, the surgical risk for meningiomas is higher in skull base. A low proliferative potential in skull base meningiomas should be taken into consideration especially when treating elderly patients with asymptomatic meningiomas $[9,16]$. 
Although there was a significant difference of the MIB-1 staining index in tumor size by chi-square test [15], tumor size is not an independent risk factor for a high MIB-1 staining index. The tumor volume is associated with the annual growth rate but not with doubling time [13, 16, 21]. Assuming that a tumor shows a constant relative volume increase, larger tumors will show higher growth rates if the annual volume increase is expressed in absolute values. Large tumors should be carefully observed even though the initial volume is not a risk factor for a high MIB-1 index, and it is not correlated with doubling time.

The etiology of peritumoral brain edema associated with meningiomas is multifactorial. Factors that may influence the etiology of peritumoral edema include tumor size, histological subtypes, vascularity, venous stasis, and brain invasion [1, 4]. Ide et al. [4] found a significant correlation of both the MIB-1 staining index and tumor size with the extent of edema. A high MIB-1 staining index itself did not seem to be directly responsible for perifocal edema, since our logistic regression analysis demonstrated that edema is not an independent risk factor for a high MIB1 index [15].

Tumors with calcification grew significantly less than those without calcification $[9,13,16]$. Absence of calcification on $\mathrm{CT}$ correlated strongly with doubling time [5]. Diffusely calcified meningiomas had a low mean MIB1 staining index of $0.57 \%$. Focally calcified tumors showed a relatively low proliferative potential $(0.92 \%)$ compared with that of noncalcified tumors $(1.75 \%)$ [15]. The results are always consistent when the relationship between calcification and proliferative potential or growth rate is compared. We also confirmed this characteristic in meningioma (OR, 0.498, $\mathrm{p}=0.019)$.

In conclusion, male, recurrence, non-skull base, absence of calcification are independent risk factors for a high proliferative potential. These should be taken into consideration when managing meningiomas.

Acknowledgements This work was supported in part by a grant-inaid for scientific research (C) from the Japanese Ministry of Education, Culture, Sports, Science, and Technology (to H. K.). We thank Mr. Takashi Sakayori and Mrs. Mihoko Kuma for their technical assistances.

\section{References}

1. Aguiar PH, Tsanaclis AM, Tella OI, Plese JP (2003) Proliferation rate of intracranial meningiomas as defined by the monoclonal antibody MIB-1. Neurosurg Rev 26:221-228

2. Cattoretti G, Becker MHG, Key G, Duchrow M, Schluter C, Galle J, Gerdes J (1992) Monoclonal antibodies against recombinant parts of the Ki-67 antigen (MIB 1 and MIB 3) detect proliferating cells in microwave-processed formalin-fixed paraffin sections. J Pathol 168:357-363
3. Ho DMT, Hsu CY, Ting LT, Chiang H (2002) Histopathology and MIB-1 labeling index predicted recurrence of meningiomas. Cancer 94:1538-1547

4. Ide M, Jimbo M, Yamamoto M, Umebara Y, Hagiwara S, Kubo O (1996) MIB-1 staining index and peritumoral brain edema of meningiomas. Cancer 78:133-143

5. Jaaskelainen J, Haltia M, Laasonen E, Wahlstrom T, Valtonen S (1985) The growth rate of intracranial meningiomas and its relation to histology. Surg Neurol 24:165-172

6. Kakinuma K, Tanaka R, Onda K, Takahashi H (1998) Proliferative potential of recurrent intracranial meningiomas as evaluated by labelling indices of BUdR and Ki-67, and tumour doubling time. Acta Neurochir 140:26-32

7. Kalala JP, Caemaert J, de Ridder L (2004) Primary resected meningiomas: relapses and proliferation markers. In vivo $18: 411-416$

8. Kayaselcuk F, Zorludemir S, Bal N, Erdogan B, Erman T (2004) The expression of surviving and $\mathrm{Ki}-67$ in meningiomas: correlation with grade and clinical outcome. J Neuro-Oncol 67:209-214

9. Kuratsu J, Kochi M, Ushio Y (2000) Incidence and clinical features of asymptomatic meningiomas. J Neurosurg 92:766-770

10. Louis DN, Scheithauer BW, Budka H, von Deimling A, Kepes JJ (2000) Meningiomas. In: Kleihues P, Cavenee WK (eds) World Health Organization classification of tumours. Pathology and genetics of tumours of the nervous system. IARCPress, Lyon, France pp176-184

11. Matsuno A, Fujimaki T, Sasaki T, Nagashima T, Ide T, Asai A, Matsuura R, Utsunomiya H, Kirino T (1996) Clinical and histopathological analysis of proliferative potentials of recurrent and non-recurrent meningiomas. Acta Neuropathol 91:504-510

12. Nakaguchi H, Fujimaki T, Matsuno A, Matsuura R, Asai A, Suzuki I, Sasaki T, Kirino T (1999) Postoperative residual tumor growth of meningioma can be predicted by MIB-1 immunohistochemistry. Cancer 85:2249-2254

13. Nakamura M, Roser F, Michel J, Jacobs C, Samii M (2003) The natural history of incidental meningiomas. Neurosurgry 53:62-71

14. Nakasu S, Hirano A, Shimura T, Llena JF (1987) Incidental meningiomas at autopsy study. Surg Neurol 27:319-322

15. Nakasu S, Nakajima M, Matsumura K, Nakasu Y, Handa J (1995) Meningioma: proliferating potential and clinicoradioloical features. Neurosurgery 37:1049-1055

16. Niiro M, Yatsushiro K, Nakamura K, Kawahara Y, Kuratsu J (2000) Natural history of elderly patients with asymptomatic meningiomas. J Neurol Neurosurg Psychiatry 68:25-28

17. Olivero WC, Lister JR, Elwood PW (1995) The natural history and growth rate of asymptomatic meningiomas: a review of 60 patients. J Neurosurg 83:222-224

18. Perry A, Stafford SL, Scheithauer BW, Suman VJ, Lohse CM (1998) The prognostic significance of MIB-1, p53, and DNA flow cytometry in completely resected primary meningiomas. Cancer 82:2262-2269

19. Roser F, Samii M, Ostertag H, Bellinzona M (2004) Clinicopathological study. The Ki-67 proliferation antigen in meningiomas. Experience in 600 cases. Acta Neurochir 146:37-44

20. Whittle IR, Smith C, Navoo P, Collie D (2004) Meningiomas. Lancet 363:1535-43

21. Yoneoka Y, Fujii Y, Tanaka R (2000) Growth of incidental meningiomas. Acta Neurochir 142:507-511

\section{Comments}

Jürgen R. E. Bohl, Mainz, Germany

The aim of this investigation on 342 patients with meningiomas was to determine the clinical and radiological features related to their proliferative capacity using the MIB-1 staining index. This seems to 
be justified as other authors had found, that in cases of meningiomas with a MIB-1 index of 3 or more percent the tumor volume doubling time was less than 2 years (see reference No. 12: Nakaguchi et al.) Therefore it would be of great benefit for all therapeuticstrategies to know some independent risk factors for a high growth rate of a meningioma prior to surgical intervention and histopathological investigation.

H. Kasuya et al. could demonstrate by using a logistic regression model that in their carefully documented series of 342 meningiomas there were four independent risk factors for a high proliferative potential. These were male, tumor recurrence, non-skull base localization and absence of calcification. Age, symptomatic behaviour, tumor volume, multiplicity and peritumoral edema proved not to be independent risk factors.

In general a lot of scientific work has been done in order to predict the outcome of a disease, especially to determine the prognosis of tumor patients. All people and institutions involved in the therapy or cure of a tumor disease want to know: How long will the patient survive?

Primarily the grading of brain tumors was based on clinical observations of survival rates and mortality diagrams. The grading of meningiomas according to the WHO-classification of brain tumors takes into consideration not only the mitotic activity and the proliferation indices but also some other histopathologic characteristics, that had proved to be important concerning the course of the disease. The survival rate was the essential criterion for evaluating the importance of many different features.

Thus it happened, that in the series of H. Kasuya et al. there were 56 of 296 grade I meningiomas with a MIB-1 staining index of greater than $3 \%$, and that in the groups of grade II and even grade III meningiomas there were two tumors ( 2 out of 46 ) with a low proliferation index (less than $3 \%$ ). Under the assumption that the grading of tumors reflects the clinical course of the disease, these observations hint to the fact, that the proliferating potential of a tumor is not the only and perhaps also not the most important property of a tumor, in concern to the outcome of the disease.

These statistically established phenomena of a tumor disease are of an inestimable value for therapeutic decisions; but we always should bear in mind, that in a very single case, in a patient just sitting in front of you, all these well known "facts" might be totally wrong and not suitable at all. Because the patient and his tumor (the tumor and its host) are performing always a very individual symbiotic process that is unique in the world.

\section{Kaoru Kurisu, Hiroshima, Japan}

The authors analyzed the relationship of MIB-1 indices to characteristics of 342 consecutive patients with meningioma surgically removed between 1995 and 2004 in Tokyo Women's Medical University by logistic regression analysis. The MIB-1 staining index in 100 of 342 meningiomas was equal to or bigger than 3.0;56 of 296 were grade I using the WHO classification, 27 of 28 grade II, 17 of 18 grade III. The authors concluded that male, recurrence, non-skull base location and absence of calcification are independent risk factors for a high proliferative potential of meningiomas from the results of MIB-1 staining of each operative specimen. This is a very clear and important conclusion. As there are in Japan many CT scanners and MRI machines and all people living in Japan have medical insurance with relatively low cost, people can be easily checked by CT or MRI when they present with minor symptoms such as slight headache or vertigo. The chance is thus high to detect meningiomas almost incidentally. Recently in Japan, it was found that meningioma is the most common primary brain tumor (Report of Brain Tumor Registry of Japan, 19691996, 11th Edition). According to the registry in 1996, the incidences of meningiomas, all gliomas, pituitary adenomas and schwannomas are $27.8 \%, 22.8 \%, 18.3 \%$ and $9.6 \%$, respectively. This tendency of incidences of each brain tumor has been lasting for several years. On the other hand, I recognize that glioma is the most common primary brain tumor in other countries in the world. Additionally, the life expectancy of Japanese people is one of the longest in the world. The rate of people older than 65 years out of the total population of Japan will be one fourth in 2015 by statistical inference. It is, therefore, important to know the natural course of each brain tumor to make enough and beneficial informed consent to the patients with brain tumors. I believe this paper provides us the proper information for patients with meningioma. Presently, we usually check patients with an incidentally found meningioma by control image after 3 months to exclude the progressive subtypes like atypical or anaplastic meningioma. 